\title{
Cirugía Robótica en Artroplastia de Rodilla
}

\section{Robotic Surgery in Knee Arthroplasty}

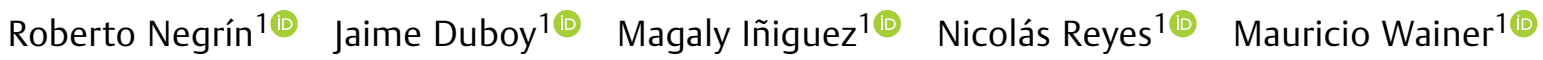 \\ Carlos Infante ${ }^{1,2}$ Gonzalo Ferrer ${ }^{1(1)}$ Nicolás Gaggero ${ }^{1,3(0)}$ Rodrigo Díaz ${ }^{1,3}$ (1)
}

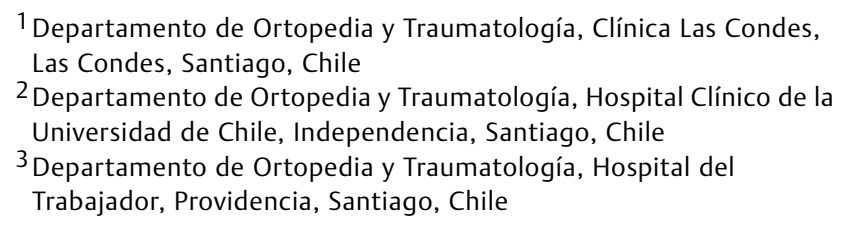

Address for correspondence Rodrigo Díaz Peraita, MD, Departamento de Ortopedia y Traumatología, Clínica Las Condes, Estoril 450, Las Condes, Santiago, Chile (e-mail: rodrigo.fdp@gmail.com).

Rev Chil Ortop Traumatol 2019;60:67-76.

\section{Resumen \\ Palabras clave \\ - Artrosis \\ - rodilla \\ - prótesis \\ - robótica \\ - precisión}

La cirugía protésica de rodilla es un procedimiento ampliamente aceptado como etapa final del tratamiento de la artrosis de rodilla, con sobrevida que supera el $90 \%$ a $10-15$ años. Dentro de las principales causas de fallo, se encuentran la infección $(20,4 \%$ y el aflojamiento mecánico (20,3\%). El uso de ayudas tecnológicas en cirugía está en constante desarrollo, con el objetivo de mejorar la precisión del acto quirúrgico. En ese escenario, la Cirugía Asistida por Computador (CAS) en artroplastia de rodilla, crece de forma exponencial, y apunta a mejorar el posicionamiento y selección del tamaño de los componentes protésicos, aumentar la precisión de las resecciones óseas y mejorar el balance de los tejidos blandos, logrando así una mayor sobrevida del implante. En comparación a las técnicas convencionales, la cirugía robótica ha mostrado mejores resultados funcionales, al primer año de seguimiento, en términos de rango articular, menor dolor post-operatorio y menor tiempo de estadía hospitalaria. Pero todavía es necesario establecer si, a largo plazo, esas diferencias funcionales se traducirán en mejores resultados clínicos que permitan, de forma consistente, inclinar la balanza en favor de la técnica asistida por robot por sobre las técnicas tradicionales.

Prosthetic knee surgery is a widely accepted procedure as the final stage in the treatment of knee osteoarthritis, with survival rate over $90 \%$ at $10-15$ years. Among the main causes of failure are infection (20.4\%) and mechanical loosening (20.3\%). The use of technological aids in surgery is in constant development, with the aim of improving the accuracy of the surgical act. In this scenario, Computer-Aided Surgery (CAS) in knee arthroplasty grows exponentially, and aims to improve the positioning and selection of the size of the prosthetic components, increase the accuracy of bone resections and improve the balance of soft tissues, thus achieving a greater survival of the implant. Compared to conventional techniques, robotic surgery has shown better functional results at the first year of follow-up, in terms of joint range, less post-operative pain and shorter hospital stay. It is still necessary to establish whether, in the long term, these functional differences will result in better clinical results that will allow - in a consistent manner - to tip the balance in favor of robot-assisted technique over traditional techniques. received

March 19, 2019

accepted

August 23, 2019
DOI https://doi.org/

10.1055/s-0039-1698417. ISSN 0716-4548.
Copyright ( 2019 by Thieme Revinter

Publicações Ltda, Rio de Janeiro, Brazil
License terms

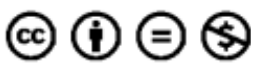




\section{Introducción}

La cirugía protésica es el tratamiento de elección en la etapa final de la artrosis de rodilla, con sobrevida global de los implantes que supera el $90 \%$ a 10-15 años según reportes. ${ }^{1,2}$ No obstante, a pesar de importantes avances en el desarrollo y tecnología aplicada a los implantes, así como también, en el perfeccionamiento en la técnica quirúrgica, la tasa de satisfacción de los pacientes no supera el $72 \%$ - 86\%. En el mismo sentido, Bhandari y col., ${ }^{3}$ describió que las tasas de revisión de Prótesis Total de Rodilla (PTR) van en constante aumento, representando una alta carga en términos de costos para los Sistemas de Salud.

En un estudio transversal reciente, que incluyó 337.597 procedimientos de revisión de PTR, Delanois R. y col., ${ }^{4}$ reportó como las causas principales de revisión a la infección (20,4\%) y el aflojamiento mecánico (20,3\%). Los principales predictores de falla secundaria a aflojamiento protésico son el mal posicionamiento de los componentes protésicos y el mal alineamiento post operatorio de la extremidad intervenida. Por esas razones, y por resultados publicados en la literatura, el enfoque actual, está dirigido al desarrollo de nuevas estrategias y tecnologías para mejorar la sobrevida de los implantes y la satisfacción de los pacientes.

La implementación de apoyo tecnológico en cirugía de reemplazo articular de rodilla está en franco aumento. La cirugía asistida por computador (CAS) es una de las que se presenta en la actualidad, ofreciendo un mejor control y flexibilidad en los movimientos quirúrgicos, y con evidencia inicial que apunta a la mejoría en la precisión del posicionamiento de implantes y así,, con el potencial de disminuir los resultados insatisfactorios.

\section{Tipos DE CAS}

Figueroa F. Y col., ${ }^{5}$ realizó una descripción detallada de los sistemas CAS y los clasificó en tres categorías principales (-Fig. 1):

- Pasivos: Corresponden a cirugías asistidas con navegación. El sistema funciona como una asistencia o guía en un procedimiento que se realiza con técnica e instrumental convencional.

- Semiactivos: Utilizan una herramienta robótica que entrega feedback instantáneo en un procedimiento realizado por un cirujano entrenado. Esa herramienta guía el nivel y orientación de corte, balance de gaps, rotación, posicionamiento y tamaño de los componentes.

- Activos: Son sistemas en que el procedimiento es llevado a cabo completamente con una herramienta robótica, sin intervención directa del cirujano.

Asimismo, se describen dos modalidades diferentes de CAS: aquella modalidad basada en imágenes, en que la planificación quirúrgica se realiza principalmente en base a imágenes (Tomografía Computarizada o Resonancia Magnética), obtenidas en la etapa pre-operatoria; y la modalidad libre de imágenes, en que los hitos anatómicos son obtenidos directamente durante la cirugía y utilizados para obtener una reconstrucción en 3D de acuerdo a la información recopilada.

Finalmente, los Sistemas de Cirugía Asistida por Robot pueden tener plataformas cerradas o abiertas, dependiendo de si aceptan exclusivamente la implantación de componentes protésicos manufacturados por una empresa específica, o bien si permiten la libertad de elección del implante de acuerdo a la preferencia del cirujano.

En el presente artículo, nos referiremos a los Sistemas de Cirugía Asistida por Computador Semiactivos, pues son los que están predominando en Cirugía Robótica en Prótesis de Rodilla. Es así como detallaremos el funcionamiento de un Sistema CAS Semiactivo, tomando como referencia a los Sistemas NAVIO (NAVIO Surgical System Smith \& Nephew, Andover, Massachusetts, USA), y MAKO (Rio Mako Stryker, Fort Lauderdale, Florida, USA), que están actualmente marcando tendencia en el mercado de la cirugía protésica y cuentan con evidencia en la literatura.

\section{¿CÓMO FUNCIONA UN SISTEMA CAS SEMIACTIVO?}

Los Sistemas de Cirugía Asistida por Computador Semiactivos se basan en una herramienta robótica

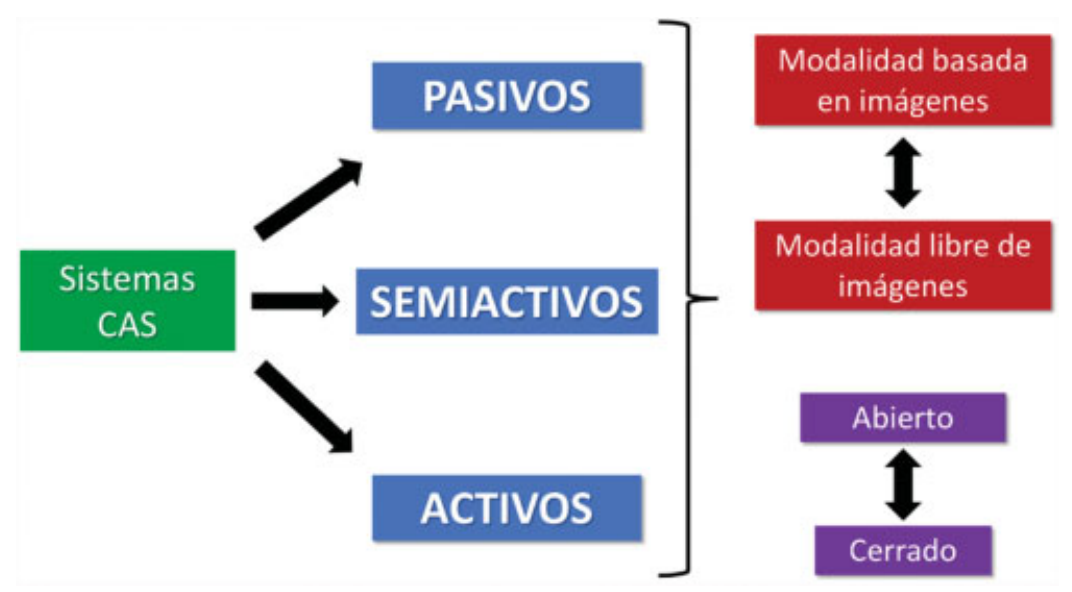

Fig. 1 Esquema de resumen de las distintas modalidades de Sistemas CAS. 


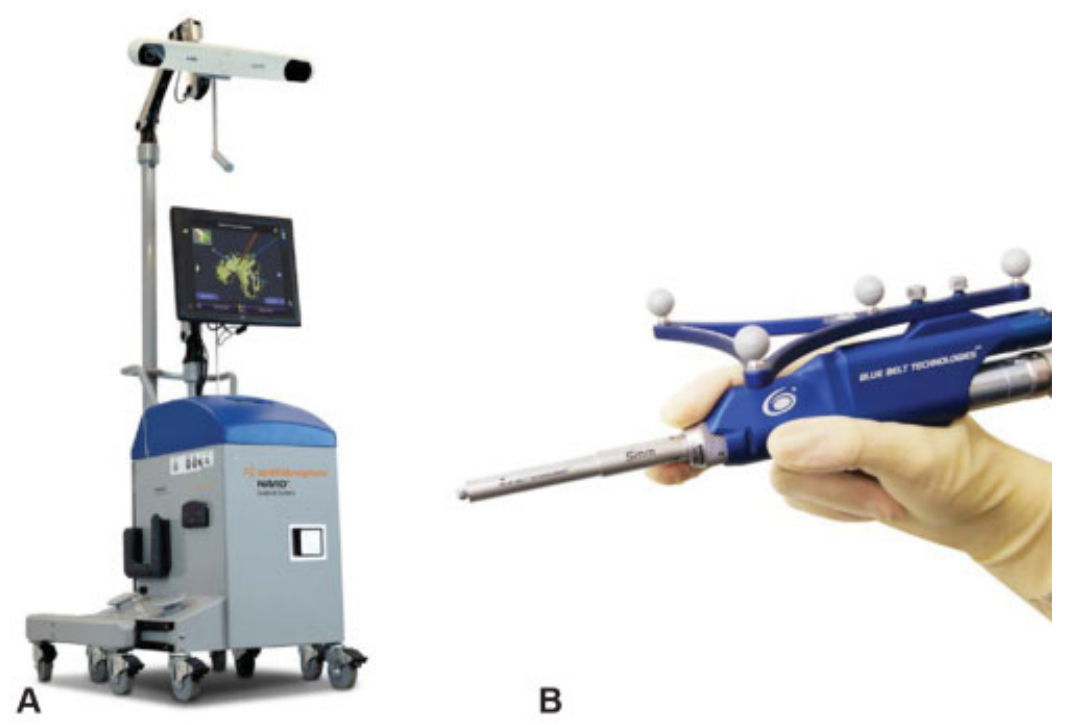

Fig. 2 Sistema quirúrgico NAVIO. (A) Consola principal; (B) Herramienta de corte manual. Extraído de: Lonner J. Robotically Assisted Unicompartmental Knee Arthroplasty with a Handheld Image-Free Sculpting Tool. Clin Orthop Relat Res., 2015.

interactiva que otorga feedback visual, de audio y/o táctil, y que de esa forma, guían la resección ósea intraoperatoria dentro de la ventana háptica predefinida. ${ }^{6}$ La acción de la fresa de corte fuera de esa ventana estereotáxica es limitada, lo que conceptualmente ayuda a preservar mayor stock óseo y a minimizar el daño a los tejidos blandos periarticulares.

\section{NAVIO (NAVIO Surgical System Smith \& Nephew, Andover, Massachusetts, USA)}

El sistema NAVIO es una herramienta de corte manual con asistencia robótica que actualmente está disponible para artroplastias de rodilla, tanto unicompartimentales como totales (-Fig. 2). La planificación de los implantes para la decisión del tamaño de los componentes, la alineación y el volumen de la extracción ósea se realiza intraoperatoriamente, sin necesidad de una Tomografía Computarizada ni Resonancia Magnética previa. ${ }^{7}$ El sistema rastrea en forma continua la posición de la extremidad inferior del paciente y el dispositivo robótico usando un sistema de navegación. Para ello se insertan pines percutáneos bicorticales en la tibia y el fémur. Se determinan intraoperatoriamente los ejes mecánicos y rotacionales de la extremidad. La anatomía condilar se traza al "mapear" las superficies con la sonda óptica. Ese proceso de registro demora aproximadamente 5 minutos en promedio. Los datos intraoperatorios luego son utilizados por los algoritmos de software del sistema para determinar los ejes óseos coronal, sagital y axial y la morfología articular.

El sistema crea un modelo virtual que le permite al cirujano seleccionar el tamaño de implante que mejor se adapta a la anatomía femoral y tibial del paciente en los planos coronal, sagital y rotacional. Los siguientes pasos se dirigen a determinar la brecha y el equilibrio de partes blandas después del posicionamiento virtual del implante, la eliminación de los osteofitos y el estrés de los ligamentos y los tejidos blandos. El sistema permite realizar una evaluación dinámica con los componentes seleccionados, graficando la tensión de los tejidos blandos en los distintos grados de rango articular. Se aplica un estrés en valgo para tensar el ligamento colateral medial (para artroplastias unicompartimentales mediales) o un estrés en varo para tensar las estructuras laterales (para artroplastias unicompartimentales laterales), las posiciones tridimensionales del fémur y la tibia se captan a través de un rango pasivo de movimiento de la rodilla ( - Fig. 3 ). Se crea una representación gráfica del espacio a través del rango de flexión y se determina si la posición planificada del componente femoral y tibial es adecuada o si requiere alguna corrección.

El Sistema NAVIO nos permite realizar ajustes modificando virtualmente tamaño, inclinación tibial, posición de implantes y profundidad de resección, para lograr el mejor equilibrio ligamentario en todo el arco del movimiento. Toda esa tecnología aplicada al procedimiento quirúrgico, permitiría una precisión mayor en el posicionamiento final de la prótesis y, por consecuencia, una mejor función y un mayor rango de movimiento post operatorio.

\section{MAKO (Rio Mako Stryker, Fort Lauderdale, Florida, USA)}

El Sistema Mako (Robotic Arm Interactive Orthopaedic System) está disponible en la práctica clínica para artroplastías unicompartimentales y totales de rodilla, y artroplastias totales de cadera. Corresponde a un sistema basado en imágenes, por tanto, requiere el uso de una Tomografía Computarizada para generar un modelo virtual 3D de la anatomía específica de cada paciente (-Fig. 4). Ese modelo virtual es ingresado al software del sistema Mako, y así, es utilizado para generar un plan pre-operatorio personalizado. ${ }^{8}$

En el intraoperatorio, el sistema Mako es utilizado para guiar la cirugía de acuerdo al plan pre-operatorio ya establecido. No obstante, ese plan puede ser ajustado 

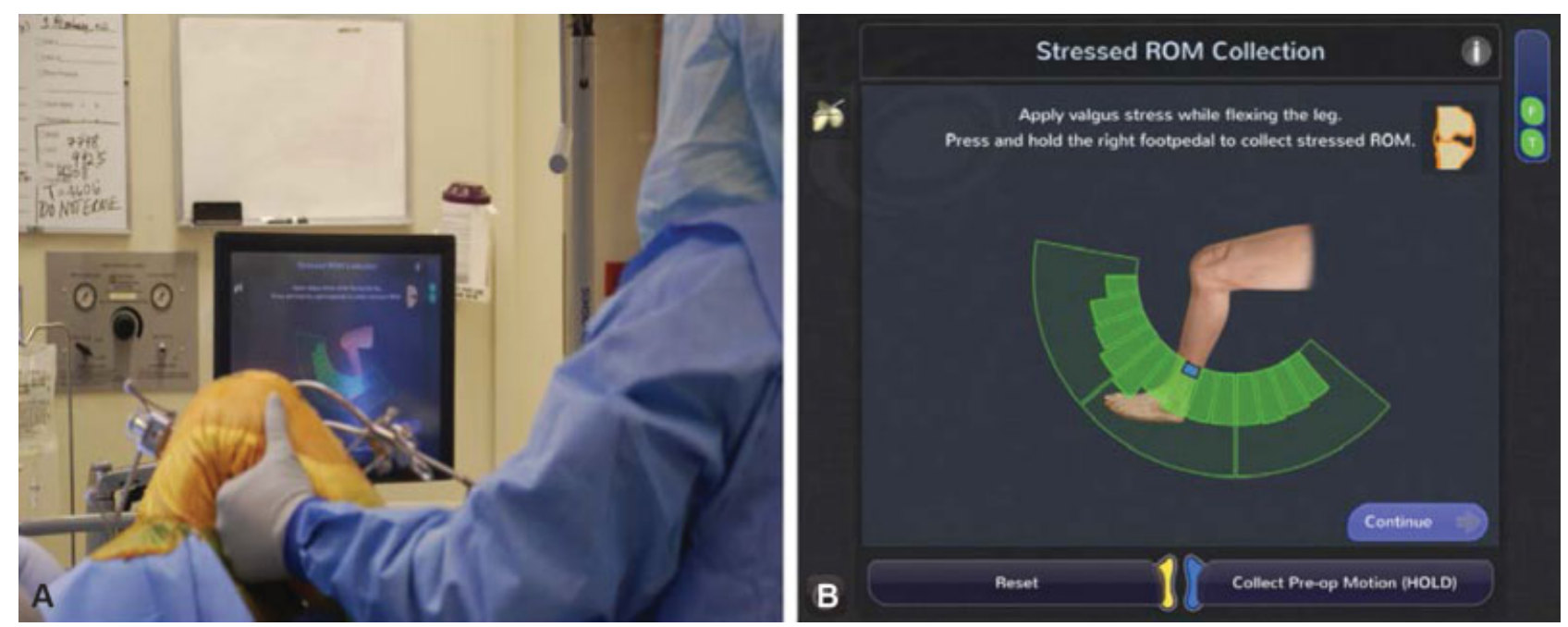

Fig. 3 Balance dinámico de partes blandas utilizando el sistema NAVIO. Registro de estrés en valgo (A y B) en artroplastia unicompartimental medial de rodilla. Extraído de: Lonner J. Robotically Assisted Unicompartmental Knee Arthroplasty with a Handheld Image-Free Sculpting Tool. Clin Orthop Relat Res., 2015.
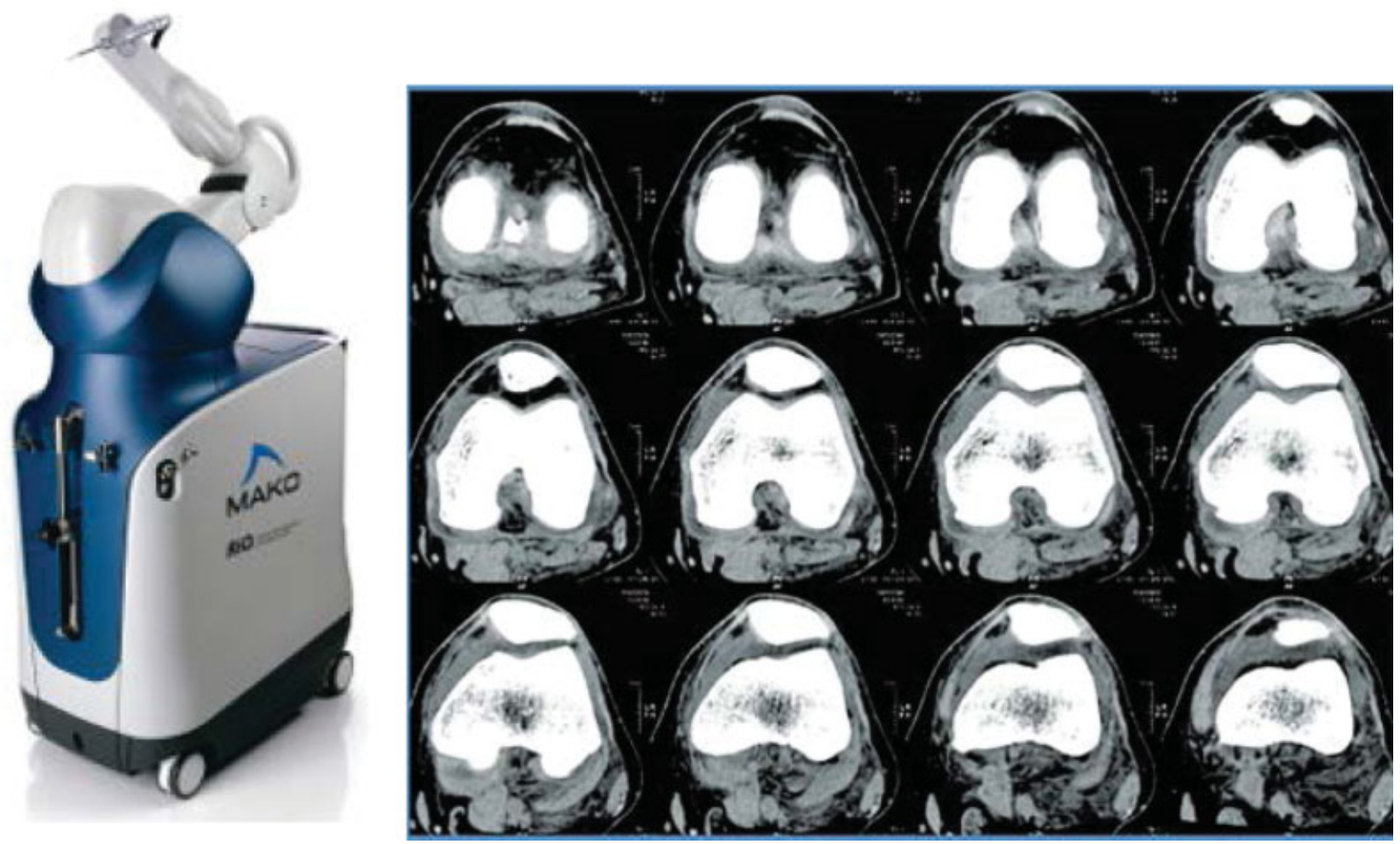

Fig. 4 Sistema robótico Mako. Con el uso de Tomografía Computarizada se genera un modelo virtual 3D de la anatomía de cada paciente. Extraído de: www.stryker.com.

durante el acto quirúrgico, dependiendo de los registros invivo, antes de hacer los cortes óseos. La herramienta de corte se encuentra adjuntada al brazo háptico, el que guiará la extensión y profundidad de resección femoral y tibial. Para las artroplastías unicompartimentales corresponde a una fresa de alta velocidad y, para las artroplastías totales, una sierra oscilante. El instrumento es guiado por el cirujano; el sistema Mako provee feedback háptico para prevenir cualquier error durante las resecciones óseas, guiando al cirujano dentro del área pre-definida y evitando el movimiento de la fresa fuera de los bordes planeados
(-Fig. 5). Ello permitiría mayor precisión en cuanto al alineamiento y posicionamiento del implante.

\section{Indicaciones y Contraindicaciones Para el uso de la técnica robótica en Artroplastias de Rodilla}

Las indicaciones para el uso de sistemas robóticos en artroplastias de rodilla siguen los mismos lineamientos que los utilizados para las técnicas convencionales. De esa forma, la artroplastia total robótica está indicada para reemplazos 


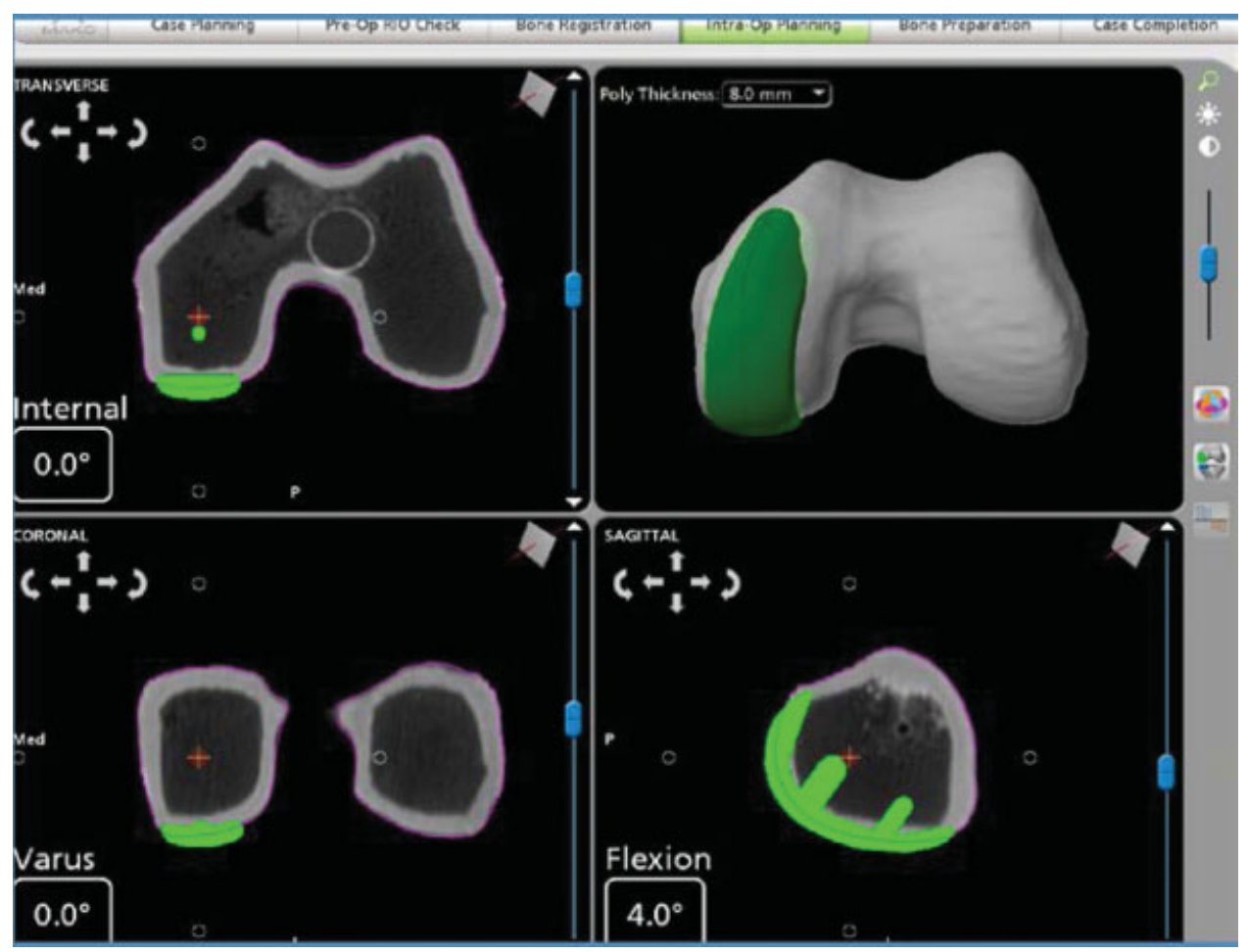

Fig. 5 Planificación virtual de resección ósea otorgado por sistema Mako para artroplastia unicompartimental de rodilla. Extraído de: www. stryker.com.

articulares primarios en pacientes con patología articular tricompartimental de rodilla secundaria a artrosis degenerativa, artrosis post-traumática, artritis reumatoide, $\mathrm{y}$ con deformidades leves a moderadas. Las contraindicaciones se circunscriben a pacientes con alguna infección activa, pacientes con enfermedades neuromusculares o mentales que pudiesen comprometer la estabilidad del implante protésico, compromiso de stock óseo que altere la adecuada fijación del implante, inmadurez esquelética, o inestabilidad severa de la rodilla. ${ }^{4,6}$

En ese sentido, la artroplastia unicompartimental robótica está indicada para pacientes con patología aislada de un solo compartimento - medial o lateral -, idealmente Ahlbäck III - IV, y con clínica concordante, es decir, sin dolor en compartimento contralateral ni patelofemoral. Otros criterios incluyen: deformidad angular $<15^{\circ}$ y reductible, contractura en flexión $<5^{\circ}$, flexión $>90^{\circ}$, y ligamentos cruzados anterior y posterior competentes. ${ }^{9}$ Contraindicaciones para la artroplastia unicompartimental son: infección activa, enfermedad articular inflamatoria, ausencia de ligamento cruzado anterior, inestabilidad ligamentaria o contractura del ligamento colateral medial, e historia de osteotomía tibial previa. ${ }^{10,11}$

\section{Cirugía Robótica vs Cirugía Convencional}

En la actualidad, existe evidencia cada vez más sólida indicando que la cirugía robótica es una herramienta que mejora, en tiempo real, la precisión en el posicionamiento y elección de tamaño del implante.

Batailler C. Y col., ${ }^{12}$ en un estudio de casos y controles utilizando el sistema NAVIO, evidenció diferencias significativas en términos de posicionamiento del componente tibial, tanto en el plano coronal como sagital; mejor alineamiento y una menor tasa de revisión, en favor de la cirugía robótica, con un seguimiento promedio de 19,7 \pm 9 meses para el grupo asistido por robot y $24,2 \pm 16$ meses para el grupo control.

Bell S. Y col, ${ }^{13}$ en un estudio de casos y controles que incluyó 120 pacientes, constató resultados similares utilizando el robot MAKO en cirugías protésicas unicompartimentales de rodilla. La cirugía robótica evidenció mejores resultados en relación al grupo convencional en cuanto a posicionamiento de los componentes protésicos femoral y tibial, en los planos coronal, sagital y axial.

Al evaluar resultados funcionales precoces y estadía hospitalaria, Haddad y col., ${ }^{14}$ demostró en una cohorte retrospectiva de 146 pacientes sometidos aleatroriamente a artroplastia total de rodilla con técnica asistida por robot o convencional, que la cirugía robótica se asociaba a menor dolor post-operatorio $(\mathrm{p}<0,001)$, menor requerimientos analgésicos $(\mathrm{p}<0,001)$, menor reducción de los niveles de hemoglobina post-operatorios ( $\mathrm{p}<0,001$ ), recuperación más precoz de la elevación activa de la extremidad con rodilla extendida, mejor rango de flexión de rodilla al alta ( $\mathrm{p}<0,001)$, y menor número de sesiones de kinesioterapia requeridos durante la etapa de rehabilitación postquirúrgica $(\mathrm{p}<0,001)$. En cuanto a la estadía hospitalaria, también hubo una disminución significativa, siendo de 77 horas (IQR 74 a 81) en cirugía robótica, comparado con 105 horas (IQR 98 a 126) en el grupo control ( $<<0.001$ ).

Iñiguez y col., ${ }^{15}$ realizó un estudio piloto experimental con piezas cadavéricas. Se aleatorizaron 26 rodillas en 2 
MDFA

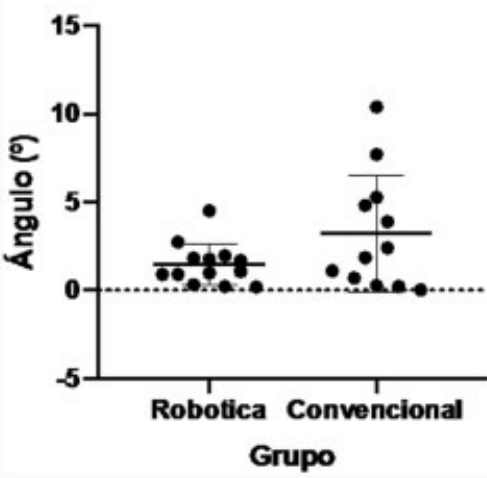

MPTA

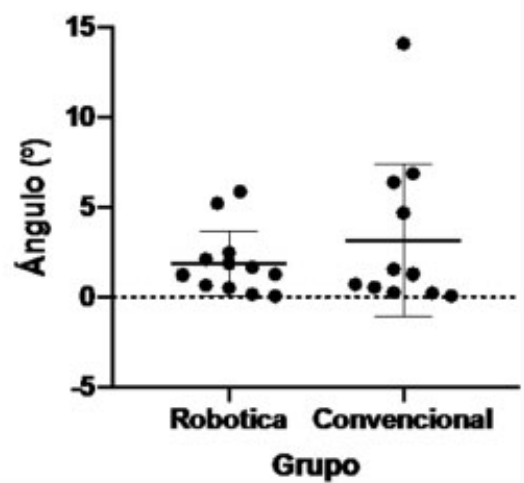

Fig. 6 Tablas de dispersión para ángulos MDFA y MPTA. Se observa una menor dispersión en el grupo de cirugía robótica versus cirugía convencional. Esta dispersión tiene un $\mathrm{p}=0,0013$ al evaluar varianzas con test de Welch. Extraído de: Iñiguez M., Negrín R., Duboy J. Robotic Assisted Unicompartmental Arthroplasty: Can We Improve Accuracy?. 12 ${ }^{\text {th }}$ Biennial ISAKOS Congress, Cancún, Mexico, 2019.

grupos para realizar cirugía protésica unicompartimental; el primer grupo (R) correspondía a cirugías asistida por robot con sistema NAVIO, y el segundo grupo (C) a cirugías con técnica convencional. Las 26 cirugías fueron realizadas por cirujanos experimentados en cirugía unicompartimental. Se realizó evaluación radiográfica, por evaluadores ciegos, pre y postoperatoria de ángulos femoral distal medial (MDFA), ángulo tibial proximal medial (MPTA), slope tibial, ángulo tibiofemoral (TFA), ángulo sagital femoral (ASF) y tamaño de componentes femoral y tibial. Al comparar ambos grupos según MDFA, se constató una mediana $1.07^{\circ}(0,19-4,5)$ para grupo $\mathrm{R}$ y $0,12^{\circ}$ para grupo $\mathrm{C}(0,03-10,4)$ con diferencia significativa en varianzas con un test de Welch con $\mathrm{p}=0,013$. En relación a MPTA, la mediana del cambio pre-post operatorio para grupo robótica y convencional, respectivamente es de $1,28^{\circ}$ $(0,05-5,87)$ y $1,3^{\circ}(0,08-14,1)(\mathrm{p}=0,3413)$ y al comparar varianzas, estas muestran un $\mathrm{p}=0,0064$, estadísticamente significativo (-Fig. 6). Al comparar los tamaños del componente femoral, se encuentra que en el grupo robótica existe un $69,23 \%$ normales, y $30,77 \%$ alterados, por su parte el grupo convencional presenta un $16,67 \%$ normal y $83,33 \%$ alterados, dando un test de Fisher con un $\mathrm{p}=0,0154$, siendo esos resultados estadísticamente significativos (-Fig. 7). Finalmente, al realizar la misma comparación con el componente tibial, no se evidenciaron diferencias significativas.

Es interesante destacar que, a pesar de las numerosas publicaciones que aportan evidencia sobre las ventajas de esa tecnología en relación a los procedimientos convencionales, la artroplastia robótica de rodilla aún no logra posicionarse como una técnica de uso masivo. Ello puede deberse a diversas razones, tales como: el costo asociado, curva de aprendizaje, incisiones adicionales para el posicionamiento de pins, mayor tiempo quirúrgico, errores en el registro de los datos, dificultades técnicas y baja evidencia de que los mejores resultados técnicos se traduzcan en mejores resultados clínicos. ${ }^{3}$

\section{Curva de Aprendizaje de Sistemas CAS}

La cirugía robótica, al igual que toda nueva técnica quirúrgica, requiere una curva de aprendizaje para lograr la destreza necesaria y así convertirla en un procedimiento

\section{Tamaño tibia}

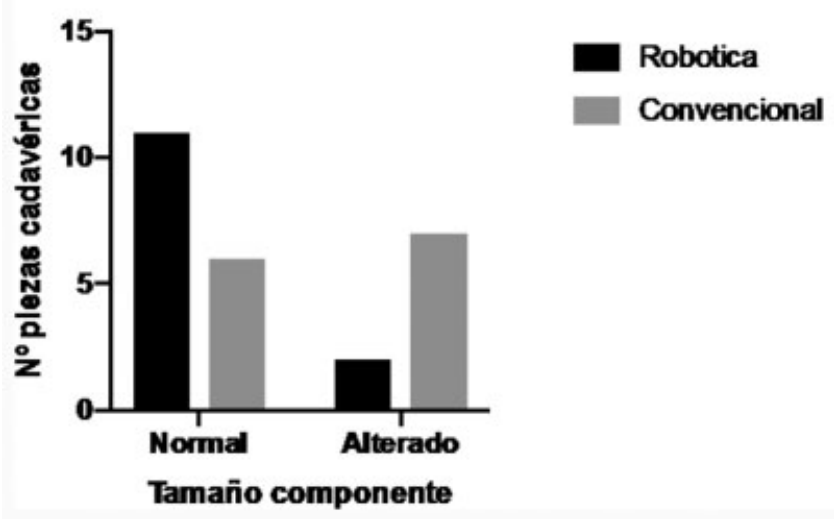

Tamaño femur

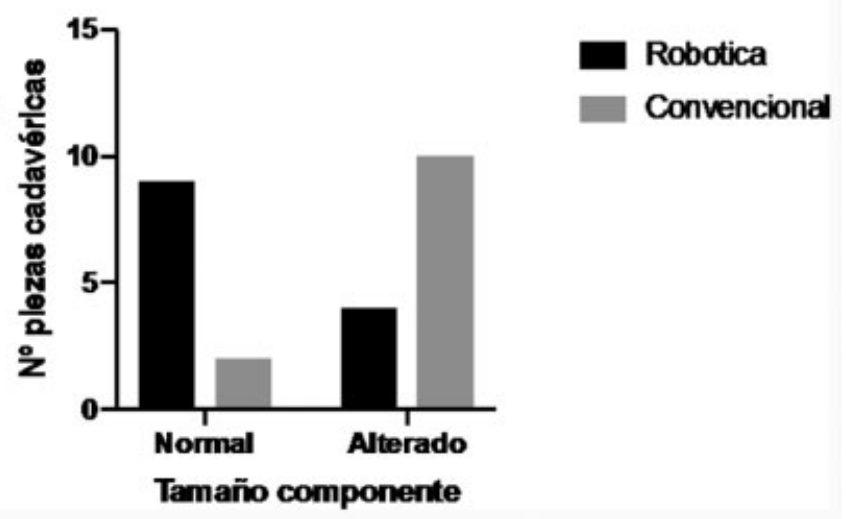

Fig. 7 Distribución según tamaño del componente tibial y del componente femoral. Se observa un claro predominio del tamaño "Normal” en la cirugía robótica. Con un $\mathrm{p}<0,05$ para el tamaño del componente femoral, pero sin diferencias para el componente tibial. Extraído de: Iñiguez M., Negrín R., Duboy J. Robotic Assisted Unicompartmental Arthroplasty: Can We Improve Accuracy?. $12^{\text {th }}$ Biennial ISAKOS Congress, Cancún, Mexico, 2019. 
eficaz y seguro. No obstante, dado la versatilidad de los diversos softwares disponibles en el mercado, es posible lograr un alto grado de competencias en un breve lapso de tiempo, incluso en el caso de cirujanos de menor experiencia. Jenny JY y col., ${ }^{16}$ en un artículo de revisión que incluyó una serie de publicaciones que analizan la curva de aprendizaje en cirugía robótica, concluyó que la cirugía protésica de rodilla asistida por robot requiere solo una breve curva de aprendizaje y, dado el constante feedback intraoperatorio durante todas las fases del procedimiento quirúrgico, permite buenos resultados incluso desde el comienzo de la experiencia en cirujanos poco entrenados.

En un estudio de cohorte prospectiva, utilizando el sistema CAS Mako RIO (robotic interactive orthopaedic system), Kayani B. Y col., ${ }^{17}$ concluyó que las artroplastias robóticas unicompartimentales se asociaban a curvas de aprendizaje de 6 casos para lograr un rendimiento óptimo en variables como el nivel de confianza del equipo quirúrgico $(\mathrm{p}<0,001)$ y los tiempos quirúrgicos ( $\mathrm{p}<0,001)$. Es importante mencionar que, en esa cohorte, la experiencia acumulativa con el uso del robot no tuvo efectos en variables como la precisión en el posicionamiento del implante $(\mathrm{p}=0,52)$, tasa de offset condilar posterior $(\mathrm{p}=0,71)$, slope tibial posterior $(\mathrm{p}=0,68)$, preservación de la línea articular nativa $(p=0,55)$, y alineamiento postoperatorio $(p=0,65){ }^{18}$

En el estudio piloto cadavérico desarrollado por nuestro grupo, analizamos la curva de aprendizaje en relación al sistema CAS NAVIO. ${ }^{15}$ Evaluamos el tiempo total del procedimiento, como reflejo del grado de confianza y destreza adquirida con el sistema, y analizamos las subvariables de tiempo de implementación, y tiempos de corte de fémur y tibia. En nuestra experiencia, se logró un plateau alrededor del quinto caso, el que se reflejó en forma proporcional en todas las variables y sub-variables evaluadas (fig. 8).

\section{¿Es la Cirugía Robótica de Rodilla Costo Efectiva?}

Ya hemos descrito las ventajas técnicas de la cirugía robótica en prótesis unicompartimental de rodilla en relación al posicionamiento de los componentes, reducción de errores en términos de alineamiento, y optimización del balance de partes blandas cuando es comparada con la técnica tradicional. ${ }^{19,20} \mathrm{Si}$ bien la evidencia existente en relación al tiempo de sobrevida de la técnica y a las tasas de revisión es aún escasa, la cirugía robótica en prótesis unicompartimental de rodilla ha demostrado tener tasas más altas de supervivencia temprana del implante en comparación con la cirugía tradicional, lo que se traduce en menores tasas de revisión. ${ }^{12}$ No obstante, persiste la duda en relación a si los beneficios reportados en la literatura justifican los costos adicionales asociados al uso de los sistemas robóticos.

Swank M. y col., ${ }^{21}$ evaluó la costo-efectividad de la tecnología de navegación aplicada a la cirugía protésica de rodilla en un estudio transversal retrospectivo, y constató que la cirugía robótica es costo-efectiva, sin aumentar la carga sobre el flujo de trabajo de un hospital ni tampoco sobre los tiempos quirúrgicos. Al aplicar los costos estimados de la cirugía robótica en relación a la artroplastia unicompartimental, demostró que incluso el costo inicial relativamente alto del sistema CAS puede ser recuperado dentro de un período de 2 años, dependiendo del flujo de pacientes, la demanda por el sistema y los resultados postquirúrgicos obtenidos. Para ese efecto, se debe asumir un incremento en el número de artroplastías robóticas unicompartimemtales y totales de 50 y 20 casos respectivamente el primer año, 70 y 30 (respectivamente) el segundo año, y 90 y 40 casos (respectivamente) en el tercer año. Asimismo, el potencial menor de tiempo de

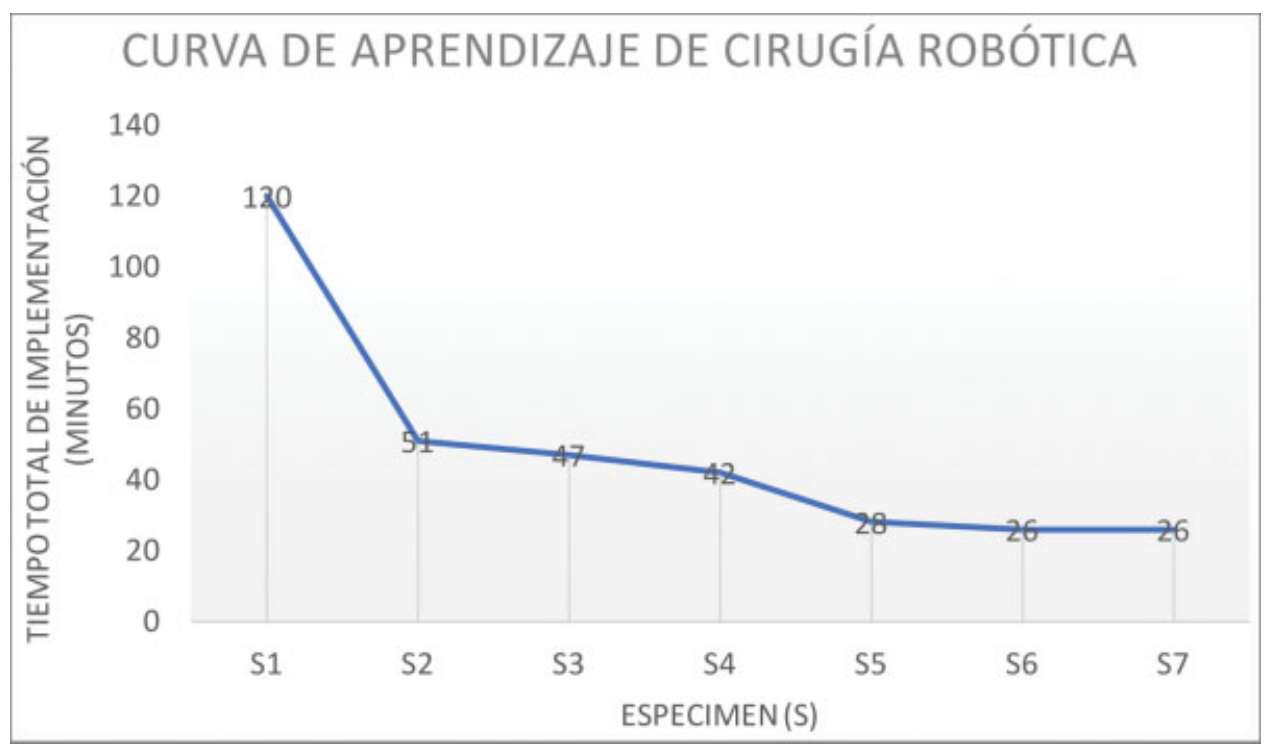

Fig. 8 Gráfico que ilustra la curva de aprendizaje de cirugía robótica en relación a tiempo total de implementación del sistema. Extraído de: Iñiguez M., Negrín R., Duboy J. Robotic Assisted Unicompartmental Arthroplasty: Can We Improve Accuracy?. $12^{\text {th }}$ Biennial ISAKOS Congress, Cancún, Mexico, 2019. 
recuperación, mayor durabilidad del implante y menores tasas de revisión obtenidas con el uso de la cirugía asistida por robot, inclinaría la balanza de costo de oportunidad en favor de ese tipo de tecnología en desmedro de técnicas convencionales.

Moschetti W. Y col., ${ }^{22}$ desarrolló un análisis de Markov para evaluar los costos, resultados, y costo-efectividad de los sistemas CAS en artroplastias unicompartimentales de rodilla. Para ello utilizó un modelo de paciente de baja demanda, edad promedio 64 años y con artrosis avanzada unicompartimental. El sistema fue costo-efectivo cuando el volumen de casos excedía 94 casos por año, la tasa de fallo a 2 años fuera menor a 1,2\%, y que el costo total del sistema fuera menor a US\$1,4 millones. Así, el número de casos por año resulta en un factor determinante para establecer la costoefectividad de los sistemas CAS.

Es necesario más estudios donde se evalúe un mayor abanico de factores que influencian el costo total del procedimiento, tales como el tiempo quirúrgico, tasa de complicaciones, tiempo de estadía hospitalaria, número de sesiones de kinesioterapia post quirúrgica, etc. Asimismo, es necesario incluir los resultados a largo plazo de la cirugía robótica y establecer si esos factores presentan alguna variación con un mayor tiempo de observación.

\section{Discusión}

La evidencia reportada en la literatura apunta a que la cirugía robótica es más precisa que las técnicas convencionales en procedimientos de artroplastias de rodilla, tanto unicompartimentales como totales. Esa mayor precisión, que se refleja en un menor número de outliers y una mayor capacidad para predecir el tamaño del componente femoral, entrega ventajas comparativas si consideramos que el malalineamiento residual y el mal posicionamiento de los componentes se encuentran dentro de las principales complicaciones asociadas a las cirugías protésicas de rodilla.

En un estudio experimental, utilizando 25 piezas cadavéricas, Lonner J. y col., ${ }^{23}$ demostró un alto grado de precisión en términos de posicionamiento del implante, utilizando el sistema CAS Navio en artroplastias unicompartimentales de rodilla. Destaca en los hallazgos de ese estudio, dispersiones significativamente menores en comparación a las técnicas convencionales en parámetros que respectan a alteraciones rotacionales y traslacionales tanto del componente femoral como del componente tibial, con promedios que fluctúan entre los $1,04^{\circ}$ a $1,88^{\circ}$ y $1,48^{\circ}$ a $1,98^{\circ}$ respectivamente. En la misma línea, esos resultados concuerdan con los hallazgos descritos en nuestro estudio experimental comparativo entre ambas técnicas, ${ }^{15}$ donde logramos demostrar la hipótesis de que existe mayor precisión en el posicionamiento de los componentes protésicos con el uso de sistemas robóticos en artroplastías unicompartimentales de rodilla. Ello se vio reflejado al comparar las varianzas en los ángulos MDFA, MPTA, Slope tibial y ASF, siendo esas diferencias significativas respecto al grupo control. Asimismo, la determinación del tamaño del componente femoral también se vio favorecida con el uso del robot. No obstante, el valor obtenido por el valor $\mathrm{p}$ en el test de Welch, no nos permite confirmar ni descartar que el sistema robótico sea superior al sistema tradicional convencional en términos de exactitud en el posicionamiento de los componentes.

El desarrollo de la cirugía robótica o de técnicas asistidas por robot en cirugía ortopédica ha hecho surgir la necesidad de establecer definiciones claras respecto a evaluaciones de “exactitud" y "precisión”. Cartiaux y col., ${ }^{24}$ basándose en un nuevo standard realizado por la International Society for Computer Assisted Orthopaedic Surgery (ISO) y la American Society for Testing and Materials (ASTM), describió la "exactitud" en cirugía ortopédica como la capacidad de lograr un resultado experimental que se acerque a un valor promedio considerado como referencial, y "precisión” como la capacidad de lograr resultados similares entre gestos quirúrgicos independientes, bajo ciertas condiciones estipuladas (-Fig. 9). En ese sentido, es posible afirmar que la cirugía robótica en prótesis de rodilla es más precisa, y por tanto más predecible, en términos de parámetros quirúrgicos objetivos en comparación a las técnicas convencionales, sin diferencias significativas en cuanto a la exactitud entre ambos procedimientos.

A pesar de las ya ampliamente descritas ventajas comparativas del uso de técnicas robóticas en artroplastias
ALTA PRECISIÓN BAJA EXACTITUD

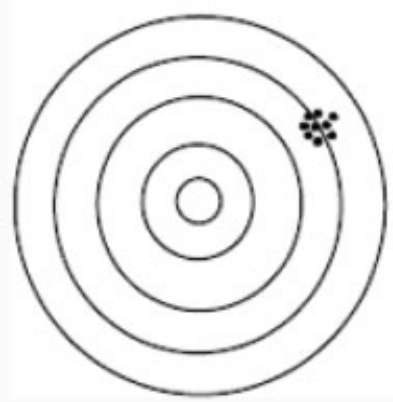

ALTA PRECISIÓN ALTA EXACTITUD

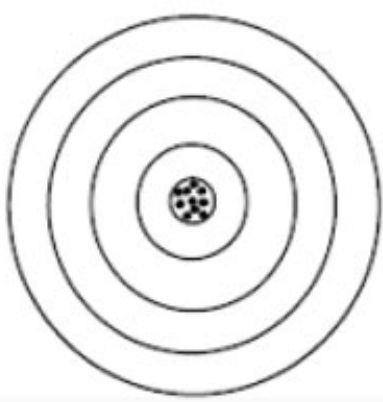

BAJA PRECISIÓN ALTA EXACTITUD

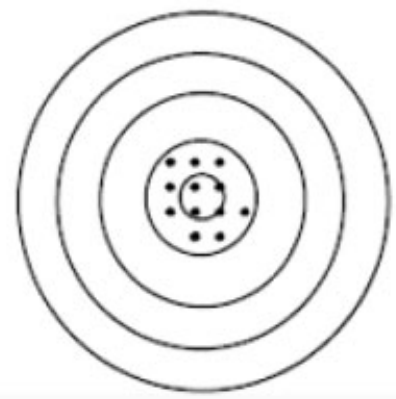

BAJA PRECISIÓN BAJA EXACTITUD

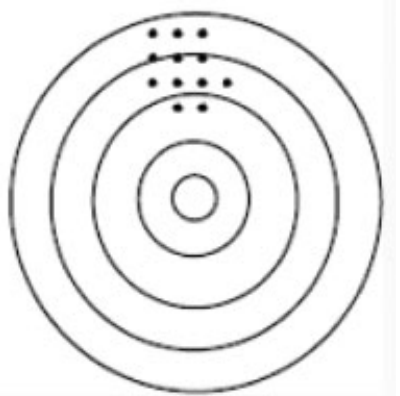

Fig. 9 Esquema descriptivo de la definición de "exactitud” (trueness) y “precisión” (precision) en relación al logro de un objetivo determinado. Adaptado de Cartiaux O., Jean-Yves J., Joscowicz L. Accuracy of Computer-Aided Techniques in Orthopaedic Surgery. How Can It Be Defined, Measured Experimentally, and Analyzed from a Clinical Perspective?. JBJS: April 19, 2017 - Volume 99 - Issue 8 - p e39. 
de rodilla, aún es controversial si esos mejores resultados técnicos se traducen a mediano y largo plazo en mejores resultados clínicos que justifiquen su elección por sobre las técnicas convencionales. Existe creciente evidencia ${ }^{14}$ acerca de la asociación entre la cirugía robótica y menor dolor post operatorio, menores requerimientos de analgésicos opioides, menor número de sesiones de kinesioterapia y mayor rango de flexión máxima al alta, en el período post quirúrgico inmediato, no obstante, existen escasos reportes en la literatura que reporten resultados a largo plazo. En un estudio controlado randomizado con un seguimiento a 2 años, Gilmour A. y col. ${ }^{25}$ reportó resultados clínicos similares en un estudio de casos y controles que incluyó 139 pacientes sometidos a artroplastias unicompartimentales robóticas versus aquellas realizadas con técnicas convencionales, sin embargo, al realizar un análisis de sub-grupo la evidencia sugiere que los pacientes más activos $(U C L A \leq 5)$ pueden beneficiarse de la cirugía robótica, con diferencias significativas en el Oxford Knee Score (OKS) $(\mathrm{p}=0,036)$. Similares resultados fueron reportados por Blyth $\mathrm{M}$. y col., ${ }^{26}$ en un estudio que incluyó 139 pacientes y un seguimiento de hasta 1 año.

\section{Conclusiones}

Con los datos obtenidos en esta revisión, podemos concluir que la cirugía robótica en artroplastias totales y unicompartimentales de rodilla, se asociaría a una mayor precisión en relación al posicionamiento de los componentes femoral y tibial, y a una mejor predicción del tamaño del componente femoral. Esa técnica ha demostrado mejorar la rehabilitación desde el post-operatorio inmediato de los pacientes, reduciendo el dolor post-operatorio, el número de sesiones de kinesioterapia y el tiempo de estadía hospitalaria, ${ }^{14}$ mejorando los resultados funcionales al primer año de seguimiento. En términos de costos asociados, el número de casos por año resulta en un factor determinante para establecer la costo-efectividad de los sistemas CAS.

Se requieren estudios a mayor escala, idealmente multicéntricos, y con un mayor tiempo de seguimiento, para establecer una comparación que nos permita concluir, con mayor evidencia, si los beneficios asociados a la cirugía robótica se traducen en mejores resultados clínicos a largo plazo.

\section{Conflicto de intereses}

Los autores declaran no tener ningún conflicto de intereses.

\section{Bibliografía}

1 Vince KG. The problem total knee replacement: systematic, comprehensive and efficient evaluation. Bone Joint J 2014;96-B (11, Supple A):105-111

2 Verra WC, van den Boom LGH, Jacobs W, Clement DJ, Wymenga $A A B$, Nelissen RGHH. Retention versus sacrifice of the posterior cruciate ligament in total knee arthroplasty for treating osteoarthritis. Cochrane Database Syst Rev 2013;(10):CD004803
3 Bhandari M, Smith J, Miller LE, Block JE. Clinical and economic burden of revision knee arthroplasty. Clin Med Insights Arthritis Musculoskelet Disord 2012;5:89-94

4 Delanois RE, Mistry JB, Gwam CU, Mohamed NS, Choksi US, Mont MA. Current Epidemiology of Revision Total Knee Arthroplasty in the United States. J Arthroplasty 2017;32(09):2663-2668

5 Figueroa F, Parker D, Fritsch B, Oussedik S. New and evolving technologies for knee arthroplasty-computer navigation and robotics: state of the art. JISAKOS 2018;3:46-54

6 Kayani B, Konan S, Tahmassebi J, Pietrzak JRT, Haddad FS. Roboticarm assisted total knee arthroplasty is associated with improved early functional recovery and reduced time to hospital discharge compared with conventional jig-based total knee arthroplasty: a prospective cohort study. Bone Joint J 2018;100-B(07):930-937

7 Lonner J. Robotically Assisted Unicompartmental Knee Arthroplasty with a Handheld Image-Free Sculpting Tool. Oper Tech Orthop 2015;25:104-113

8 Marchand RC, Sodhi N, Bhowmik-Stoker M, et al. Does the Robotic Arm and Preoperative CT Planning Help with 3D Intraoperative Total Knee Arthroplasty Planning? J Knee Surg 2019;32(08): 742-749

9 Christ AB, Pearle AD, Mayman DJ, Haas SB. Robotic-Assisted Unicompartmental Knee Arthroplasty: State-of-the Art and Review of the Literature. JArthroplasty 2018;33(07): 1994-2001

10 Campi S, Tibrewal S, Cuthbert R, Tibrewal SB. Unicompartmental knee replacement - Current perspectives. JClin Orthop Trauma 2018;9(01):17-23

11 Goodfellow J, OC J, Dodd CAF, Murray DW. Unicompartmental Arthroplasty with the Oxford Knee. New York: Oxford University Press; 2006

12 Batailler C, White N, Ranaldi FM, Neyret P, Servien E, Lustig S. Improved implant position and lower revision rate with roboticassisted unicompartmental knee arthroplasty. Knee Surg Sports Traumatol Arthrosc 2019;27(04):1232-1240

13 Bell SW, Anthony I, Jones B, MacLean A, Rowe P, Blyth M. Improved accuracy of component positioning with robotic-assisted unicompartmental knee arthroplasty: data from a prospective, randomized controlled study. J Bone Joint Surg Am 2016;98(08): 627-635

14 Kayani B, Konan S, Tahmassebi J, Rowan FE, Haddad FS. An assessment of early functional rehabilitation and hospital discharge in conventional versus robotic-arm assisted unicompartmental knee arthroplasty: a prospective cohort study. Bone Joint J 2019;101-B(01):24-33

15 Iñiguez M, Negrín R, Duboy J Robotic Assisted Unicompartmental Arthroplasty: Can We Improve Accuracy? $12^{\text {th }}$ Biennial ISAKOS Congress, Cancún, Mexico, 2019

16 Jenny JY, Picard F. Learning navigation - Learning with navigation. A review. SICOT J 2017;3:39

17 Kayani B, Konan S, Pietrzak JRT, Huq SS, Tahmassebi J, Haddad FS. The learning curve associated with robotic-arm assisted unicompartmental knee arthroplasty: a prospective cohort study. Bone Joint J 2018;100-B(08):1033-1042

18 Kayani B, Konan S, Huq SS, Tahmassebi J, Haddad FS. Robotic-arm assisted total knee arthroplasty has a learning curve of seven cases for integration into the surgical workflow but no learning curve effect for accuracy of implant positioning. Knee Surg Sports Traumatol Arthrosc 2019;27(04):1132-1141

19 Lonner JH, John TK, Conditt MA. Robotic arm-assisted UKA improves tibial component alignment: a pilot study. Clin Orthop Relat Res 2010;468(01):141-146

20 Plate JF, Mofidi A, Mannava S, et al. Achieving accurate ligament balancing using robotic-assisted unicompartmental knee arthroplasty. Adv Orthop 2013;2013:837167

21 Swank ML, Alkire M, Conditt M, Lonner JH. Technology and costeffectiveness in knee arthroplasty: computer navigation and robotics. Am J Orthop 2009;38(2, Suppl):32-36 
22 Moschetti WE, Konopka JF, Rubash HE, Genuario JW. Can RobotAssisted Unicompartmental Knee Arthroplasty Be Cost-Effective? A Markov Decision Analysis. J Arthroplasty 2016;31(04):759-765

23 Lonner JH, Smith JR, Picard F, Hamlin B, Rowe PJ, Riches PE. High degree of accuracy of a novel image-free handheld robot for unicondylar knee arthroplasty in a cadaveric study. Clin Orthop Relat Res 2015;473(01):206-212

24 Cartiaux O, Jean-Yves J, Joscowicz L. Accuracy of Computer-Aided Techniques in Orthopaedic Surgery. How Can It Be Defined,
Measured Experimentally, and Analyzed from a Clinical Perspective? J Bone Joint Surg Am 2017;99(08):e39

25 Gilmour A, MacLean AD, Rowe PJ, et al. Robotic-Arm-Assisted vs Conventional Unicompartmental Knee Arthroplasty. The 2-Year Clinical Outcomes of a Randomized Controlled Trial. JArthroplasty 2018;33(7S):S109-S115

26 Blyth MJG, Anthony I, Rowe P, Banger MS, MacLean A, Jones B. Robotic arm-assisted versus conventional unicompartmental knee arthroplasty. Bone Joint Res 2017;6(11):631-639 